\title{
The influence of Tongqiao Huashuan Decoction on the expression of VEGF and EphB2 in MCAO model rats
}

\author{
Jing $\mathrm{Cai}^{1}$, Chunlan $\mathrm{Wu}^{2}$, Chaozheng Tang ${ }^{3}$, Jin $\mathrm{Cai}^{4}$, Peng $\mathrm{Cao}^{5}$, Guangqi $\mathrm{Zhu}^{1}$
}

${ }^{1}$ Department of Neurology, ${ }^{2}$ Department of Education, The First Affiliated Hospital of Guizhou University of Traditional Chinese Medicine, Guiyang 550001, China; ${ }^{3}$ Department of Geriatrics, Leshan Hospital of Traditional Chinese Medicine, Leshan 614000, China; ${ }^{4}$ Department of Pediatrics, ${ }^{5}$ Laboratory Department of Specialty, The First Affiliated Hospital of Guizhou University of Traditional Chinese Medicine, Guiyang 550001, China Contributions: (I) Conception and design: J Cai, G Zhu; (II) Administrative support: G Zhu; (III) Provision of study materials or patients: C Tang, J Cai, P Cao; (IV) Collection and assembly of data: J Cai, C Wu; (V) Data analysis and interpretation: J Cai, C Wu, G Zhu; (VI) Manuscript writing: All authors; (VII) Final approval of manuscript: All authors.

Correspondence to: Guangqi Zhu. Department of Neurology, The First Affiliated Hospital of Guizhou University of Traditional Chinese Medicine, Guiyang 550001, China. Email: zhuguangqii@163.com.

Background: To observe the effect of Miao medicine and Tongqiao Huashuan Decoction on the expression of vascular endothelial growth factor (VEGF) and ephrin-B2 (EphB2) in the frontal lobe of the involved side and the cerebellum of rat models of middle cerebral artery occlusion (MCAO) and to reveal the pharmacological mechanism of Tongqiao Huashuan Decoction in treating acute ischemic stroke.

Methods: Seventy healthy male SD rats were randomly divided into sham operation, model, salvia miltiorrbiza (S. miltiorrhiza), and Miao medicine groups. Modified Longa's method was used to prepare a cerebral ischemia reperfusion model. After the operation, the rats in the sham operation group and model group were intragastrically administered with saline, those in the Miao medicine group were intragastrically administered with Tongqiao Huashuan Decoction, and those in the S. miltiorrbiza group were intraperitoneally injected with $S$. miltiorrbiza. After 14 days of administration, the neurological deficit scores of the rats in each group were compared before and after treatment. Hematoxylin-eosin (HE) staining was used to observe the pathological changes of the brain tissues in the right infarcted areas of the rats. VEGF expression in the frontal lobe and cerebellum was observed through immunohistochemistry, and in situ hybridization to detect EphB2 expression in the frontal lobe and cerebellum.

Results: The neurological deficit scores were significantly improved in the Miao medicine and $S$. miltiorrbiza groups aftertreatment compared with those of the model group $(\mathrm{P}<0.05)$ and was higher in the Miao medicine group than in the S. miltiorrbiza group. The VEGF expression in the right frontal lobe and cerebellum was significantly increased in the Miao medicine and $S$. miltiorrbiza groups $(\mathrm{P}<0.05)$ with the former having higher levels than the latter $(\mathrm{P}<0.05)$. EphB2 expression was significantly increased in the frontal lobe and cerebellum in the Miao medicine and S. miltiorrbiza groups $(\mathrm{P}<0.05)$ and was higher in the frontal lobe of the Miao medicine group than that of the $S$. miltiorrbiza group $(\mathrm{P}<0.05)$ but was not significantly different in the cerebellum in the $S$. miltiorrbiza and Miao medicine groups $(\mathrm{P}>0.05)$.

Conclusions: Tongqiao Huashuan Decoction can improve the neurological function score and promote the VEGF expression in the frontal lobe and cerebellum and the EphB2 expression in the frontal lobe of the involved side of MCAO rats. The pharmacological mechanism of Tongqiao Huashuan Decoction in treating acute ischemic stroke may be related to its regulation of VEDF and EphB2 expression in the distal part of the involved side.

Keywords: Miao medicine; Tongqiao Huashuan Decoction; middle cerebral artery occlusion (MCAO); vascular endothelial growth factor (VEGF); ephrin-B2 (EphB2)

Submitted Sep 04, 2019. Accepted for publication Nov 05, 2019.

doi: 10.21037/apm.2019.11.09

View this article at: http://dx.doi.org/10.21037/apm.2019.11.09 


\section{Introduction}

Ischemic stroke belongs to the category of "apoplexia" in traditional Chinese medicine and accounts for approximately $70-80 \%$ of total cerebral stroke cases. Studies worldwide have investigated ischemic brain injuries from the cellular level to the genetic level, including inflammatory response, free radicals, excitatory amino acids, apoptosis, and nerve axon regeneration. Given that the central nervous system is an integral network structure, the structure and function of its related parts are also affected when any of its parts is damaged. Following unilateral cerebral cortex infarction, secondary degenerative atrophy occurs in the striatum, thalamus, and substantia nigra of the infarcted side of the brain. Nerve connection and information transmission between the cortex and these distant parts are disturbed successively, and this condition may manifest as hyperactivity or inhibition, indicating that injuries caused by cerebral infarction are not limited to the infarcted center and penumbra and also affect the distant parts $(1,2)$.

Secondary injuries of distant parts of cerebral infarction have been widely investigated in China. The factors involved in the secondary injuries of the distant parts of a cerebral infarction include increased nerve growth inhibitor, neurotrophic disorder, decreased regional cerebral blood flow, inflammation, degenerative changes of axon, inhibition of protein synthesis, and imbalanced neurotransmitter regulation. Cerebral ischemia can induce angiogenesis in local tissues and involves the inducer of angiogenesis vascular endothelial growth factor (VEGF), which actively participates in and inhibit apoptosis. Ephrin-B2 (EphB2) is a member of tyrosine protein kinase receptor family that can promote brain neovascularization after cerebral infarction (3-7).

In modern traditional Chinese medicine, apoplexy is treated with many prescriptions and medicines. The origin of Miao medicine has a long history but has not been documented. The inheritance of Miao medicine only depends on oral transmission, forming a theoretical model system of "outline, meridian, symptoms and diseases" of Miao medicine. In the theory of Miao medicine, diseases with acute onset, dangerous condition, convulsion, coma, and other symptoms similar to stroke are classified as meridian syndrome. Miao doctors ignore that "tendon is airway and pulse is blood path". When tendons are damaged, Qi is blocked, and promoting the normal blood circulation is difficult, leading to blood stasis. Therefore, the principal treatment for impotence and weakness, dyskinesia or numbness, and other pathological changes of the limbs in Miao medicine involves the promotion of blood circulation and removal of blood stasis. Tongqiao Huashuan Decoction, which was prepared by Professor Zhu Guangqi on the basis of folk collection and clinical observation in Miao area, is an effective prescription of Miao medicine (8-10) for treating apoplexy. This experimental study explores the mechanism of Tongqiao Huashuan Decoction in improving the neurological deficit of middle cerebral artery occlusion (MCAO) rats by evaluating its effect on the expression of VEGF and EphB2 in the frontal lobe and the cerebellum of the involved side. This study aims to provide a Miao prescription with remarkable curative effect for the treatment of ischemic stroke.

\section{Methods}

\section{Laboratory animals and grouping}

Seventy healthy male SD rats aged 3 months, clean grade, and weighing 250-280 g were provided by Guizhou Medical University Laboratory Animal Center, Animal Qualification Certificate No. SCXK (Guizhou 2015-0006). They were fed adaptively for 1 week under the same light and humidity conditions and then freely fed with standard feed provided by Guizhou Medical University Laboratory Animal Center and provided with clean tap water. Indoor temperature was maintained within $15-18{ }^{\circ} \mathrm{C}$, and indoor humidity was approximately $70 \%$. The rats were fasted for $24 \mathrm{~h}$ before operation but freely provided with water. Sixteen rats randomly selected from the 70 rats were enrolled into the sham operation group. The 54 remaining rats were subjected to MCAO. The successfully modeled rats were randomly divided into three groups: model, Miao medicine, and salvia miltiorrbiza (S. miltiorrbiza) groups. This study was approved by the animal care welfare committee of Guizhou University of Traditional Chinese Medicine (ID: 20190001).

\section{Experimental drugs}

Tongqiao Huashuan Decoction consists of sargentgloryvine stem $15 \mathrm{~g}$, spinyleaf pricklyash root $15 \mathrm{~g}$, birudo $6 \mathrm{~g}$, aleppo dock rbizome $12 \mathrm{~g}$, rbizoma cibotii $12 \mathrm{~g}$, rbizoma calami $10 \mathrm{~g}$, gastrodiae rbizoma $10 \mathrm{~g}$, and astragalus membranaceus $15 \mathrm{~g}$. All of the drugs were provided by the Chinese Medicine Room of the First Affiliated Hospital of Guizhou University 
of Traditional Chinese Medicine. Decoction method: after 60 min of soaking in sufficient water, the original drugs with the water were decocted in a Chinese herbal medicine decoction machine for $30 \mathrm{~min}$. The processes were repeated three times. The decocting solution was pooled together, added into an electrothermal constant temperature water tank, and concentrated to $1.5 \mathrm{~g}$ of the original drugs per $\mathrm{mL}$. The samples were sealed and stored in a refrigerator at $4^{\circ} \mathrm{C}$. The validity of the liquid was set to 1 week and was prepared weekly. Compound danshen injection was provided by Shanghai General Pharmaceutical Co., Ltd. (Shanghai, China).

\section{Main instruments and reagents}

The materials and instruments included the Immunohistochemical VEGF kit (Beijing Zhongshan Biotechnology Company, Beijing, China), in situ hybridization detection kit of EphB2 (Nanjing Jiancheng Bioengineering Institute, Nanjing, China), electric-heated thermostatic water bath (Shanghai Medical Thermostatic Equipment Factory, Shanghai, China), paraffin microtome (German MICROM HM325, Germany), microscope (Japanese OLYMPUS BX-60, Japan), Biomias 99 image analysis system (Institute of Image and Graphics, Sichuan University, Chengdu, China), and HIPAS1000 computer high-definition color image analyzer (produced by Qianping Image Company, Tongji Medical College, Huazhong University of Science and Technology, Wuhan, China).

\section{Establishment of experimental animal model of cerebral ischemia reperfusion (MCAO)}

Longa's thread embolization method was used to prepare a cerebral ischemia rat model (11). The sham operation group underwent the same procedure, except that nylon thread insertion was not performed. At $2 \mathrm{~h}$ after the operation, the neurological status of the rats was assessed immediately after awakening according to Zea-Longa's neurological deficit score. The grades indicated the following: grade I, 0: no symptoms of neurological deficits; grade II, 1: mild neurological deficits, with left forepaw failing to extend fully; grade III, 2: moderate neurological deficits, circling left while walking; grade IV, 3: severe neurological deficits, toppling over left while walking; grade V, 4: decreased level of consciousness, failing to walk spontaneously. The rats with grades II to IV (1-3 points) in the neurological function assessment were included as successful models in the study. The rats with grades less than II and the rats that died during surgery were excluded.

\section{Treatment}

After successful modeling, the rats in each group were treated at $6 \mathrm{~h}$ after operation. The rats in the sham operation group and model group were intragastrically administered with saline. The rats in the $S$. miltiorrbiza group were given $S$. miltiorrbiza injections $(1.78 \mathrm{~mL} / \mathrm{kg} / \mathrm{day}$, once a day), and the rats in the Miao medicine group were intragastrically administered with Tongqiao Huashuan Decoction ( $3 \mathrm{~mL} /$ day) for a total of 14 days.

\section{Sample collection and detection}

Before the rats were executed, the neurological functions of the rats were assessed according to Zea-Longa's level $\mathrm{V}$ scoring standard. Brain tissues were collected and fixed in $4 \%$ paraformaldehyde. Half of the specimens were randomly selected from each group and fixed in 4\% paraformaldehyde for $24 \mathrm{~h}$. The brain tissues were embedded in paraffin and cut into two parts in the coronal plane at $2 \mathrm{~mm}$ in front and behind the optic chiasm. The specimens were sectioned (3-5 $\mu \mathrm{m})$ and used for the hematoxylin-eosin $(\mathrm{HE})$ staining of the cerebral tissues of MCAO-involved side. VEGF expression in the frontal lobe and cerebellum were immunohistochemically detected in accordance with the manufacturer's instructions. The remaining brain specimens of each group were placed in $4 \%$ paraformaldehyde for $30 \mathrm{~min}$ and then in $30 \%$ sucrose overnight until the specimen sank to the bottom. The brain specimens were cut in the coronary plane into two parts at $2 \mathrm{~mm}$ in front and behind the optic chiasm. Frozen sections $(5 \mu \mathrm{m})$ were prepared, allowed to dry in air, and placed in a refrigerator at $-40{ }^{\circ} \mathrm{C}$ for the detection of in situ EphB2 hybridization of the frontal lobe of MCAO-involved side and the cerebellum according to the manufacturer's instructions. The positive cells of VEGF and EphB2 appeared brown. Under the microscope at 400× magnification, five nonoverlapping views each section was randomly observed. The number of positive expression was counted and converted into the number of positive cells $/ \mathrm{mm}^{2}$ for evaluation. During the analysis, the magnification of all 
Table 1 Neurological deficit scores of rats before and after treatment $(\bar{x} \pm \mathrm{s})$

\begin{tabular}{lll}
\hline Group & Before treatment & After treatment \\
\hline $\begin{array}{l}\text { Sham operation group } \\
(n=16)^{*}\end{array}$ & $0.00 \pm 0.000^{*}$ & $0.00 \pm 0.000^{*}$ \\
Model group $(n=16)$ & $2.56 \pm 0.629^{*}$ & $2.50 \pm 0.632^{*}$ \\
S. miltiorrhiza group $(n=16)$ & $2.44 \pm 0.512^{*}$ & $1.94 \pm 0.443^{\star \#}$ \\
Miao medicine group $(n=16)$ & $2.51 \pm 0.627^{\star}$ & $1.31 \pm 0.479^{\star \sharp \Delta}$
\end{tabular}

Compared with the sham operation group, * $\mathrm{P}<0.05$; compared with the model group, ${ }^{\#,}, \mathrm{P}<0.05$; compared with the $S$. miltiorrhiza group, ${ }^{\triangle}, \mathrm{P}<0.05$. S. miltiorrhiza, salvia miltiorrhiza.

sections and the light source intensity were the same.

\section{Statistical methods}

SPSS 23.0 software was used to analyze final data statistics, and measurement data were expressed as mean \pm standard deviation $(\bar{x} \pm s)$. For data satisfying normal distribution, homogeneity test of variance was first conducted. One-way analysis of variance (ANOVA) was used when data satisfied the homogeneity of variance, and least significant difference (LSD) was performed to compare the paired variates. If the variance of data was not homogeneous, then Welch test was used. Dunnett's T3 method was used to compare the paired variates. Significant difference, extremely significant difference, and no statistical significance are indicated at $\mathrm{P}<0.05, \mathrm{P}<0.01$, and $\mathrm{P}>0.05$, respectively.

\section{Results}

\section{Model preparation}

Seventy rats were used in the study, including 16 in the sham operation group and 54 in the MCAO group. Six rats were excluded because of death or decreased consciousness level following the operation, showing a success rate of $88.89 \%$. No death was observed in the sham operation group or the successfully-modeled rats in the 2 weeks of treatment. The wounds of the rats healed well after operation in all of the groups. No bleeding, exudation, or infection occurred. In the sham operation group, no evident abnormalities in spirit, behavior, activity, and diet and neurological dysfunction were observed. In the MCAO group, the rats suffered from mental depression, inability to move, reduced activity, and reduced water intake. They also showed Horner's sign; subsided right eyeball, increased eye secretion, deviation of head to the left, left forelimb drooping weakness, evident circling of the left foot while walking, tilting to the left, and body bending to the left when the tail was lifted after awakening.

\section{Neurological deficit scoring of rats after treatment}

Table 1 shows the neurological deficit scores of the rats in each group. Before the treatment, the neurological deficit scores of the model, Miao medicine, and S. miltiorrbiza groups increased significantly $(\mathrm{P}<0.05)$ compared with that of the sham operation group. However, no significant difference was found among the three groups $(\mathrm{P}>0.05)$.

After the treatment, no significant difference was observed in neurological deficit scores between the sham operation and model groups $(\mathrm{P}>0.05)$. The neurological deficit scores of the Miao medicine and S. miltiorrbiza groups decreased significantly compared with those before treatment $(\mathrm{P}<0.05)$. Compared with those of the model group, the neurological deficit scores of the Miao medicine and S. miltiorrbiza groups decreased significantly $(\mathrm{P}<0.05)$. Compared with those of the S. miltiorrbiza group, the scores of neurological deficit in the Miao medicine group decreased significantly $(\mathrm{P}<0.05)$.

\section{Pathological examination with HE staining}

The right cerebral cortex of the rats in the sham operation group had normal structure, showing dense stroma without edema, clear outline and normal morphology of brain cells without solid shrinkage or dissolution, and clear nucleoli. In the model group, the right brain of the rats had marked degenerative changes. The cortical cell membrane was unclear; pyknosis, deformation, and strong basophilic change were observed in some of the neurons; and cytoplasmic deep dyeing occurred even when numerous neurons being lost, resulting in large vacuoles. The neurons showed degeneration, necrosis, and occasional nuclear pyknosis but interlaced with normal neurons in the Miao medicine group and the $S$. miltiorrbiza group and were alleviated in varying degrees compared with those of the 

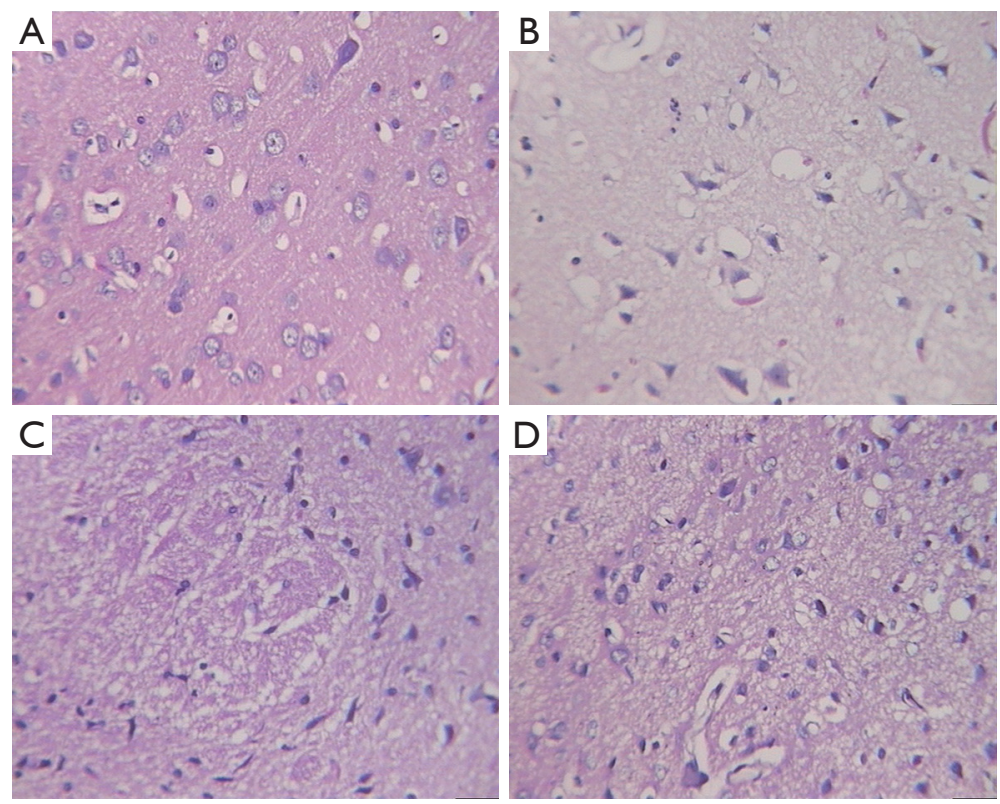

Figure $1 \mathrm{HE}$ staining of brain pathological sections of the MCAO side of rats (HE, $\times 400)$. (A) Sham operation group; (B) model group; (C) S. miltiorrbiza group; (D) Miao medicine group. HE, hematoxylin-eosin; MCAO, middle cerebral artery occlusion; S. miltiorrbiza, salvia miltiorrbiza.

Table 2 VEGF (number/ $/ \mathrm{mm}^{2}$ ) expression in the frontal lobe and cerebellum of rats in all groups $(\bar{x} \pm \mathrm{s})$

\begin{tabular}{lll}
\hline Groups & Lobe & Cerebellum \\
\hline Sham operation group $(n=8)$ & $6.75 \pm 2.053$ & $2.25 \pm 1.282$ \\
Model group $(n=8)$ & $9.38 \pm 1.506^{*}$ & $8.50 \pm 1.309^{\star}$ \\
S. miltiorrhiza group $(n=8)$ & $12.00 \pm 2.268^{\star \#}$ & $24.38 \pm 7.190^{* \#}$ \\
Miao medicine group $(n=8)$ & $15.00 \pm 3.024^{* \# \Delta}$ & $76.25 \pm 9.618^{* \# \Delta}$ \\
\hline
\end{tabular}

Compared with the sham operation group, *, $\mathrm{P}<0.05$; compared with the model group, \#, $\mathrm{P}<0.05$; compared with the $S$. miltiorrhiza group, ${ }^{\triangle}, \mathrm{P}<0.05$. VEGF, vascular endothelial growth factor; $S$. miltiorrhiza, salvia miltiorrhiza.

model group (Figure 1).

$V E G F$ expression in the frontal lobe and cerebellum of the right brain in Miao medicine group and S. miltiorrhiza group

In the frontal lobe and cerebellum of the rats, the levels of VEGF were significantly higher in the Miao medicine and S. miltiorrbiza groups compared with those in the sham operation and model groups $(\mathrm{P}<0.05)$, significantly higher in the Miao medicine and the $S$. miltiorrbiza group compared with those in the model group $(\mathrm{P}<0.05)$, and significantly higher in the Miao medicine group compared with those in the $S$. miltiorrbiza group $(\mathrm{P}<0.05)$. The results are shown in Table 2 and Figure 2.

\section{EphB2 expression in the frontal lobe and cerebellum of rats in the Miao medicine group and S. miltiorrbiza group}

In the frontal lobe and cerebellum of the rats, EphB2 levels were significantly lower in the model group compared with those in the sham operation group $(\mathrm{P}<0.05)$, significantly higher in the Miao medicine and S. miltiorrbiza groups compared with those in the sham operation group $(\mathrm{P}<0.05)$, and significantly higher in the Miao and S. miltiorrbiza groups compared with those in the model group $(\mathrm{P}<0.05)$. In the frontal lobe of the rats, EphB2 levels were significantly higher in the Miao medicine group compared with those in the $S$. miltiorrbiza group $(\mathrm{P}<0.05)$. In the cerebellum of the rats, no significant difference was found between the EphB2 levels in the S. miltiorrhiza and Miao medicine groups $(\mathrm{P}>0.05)$. The results are shown in Table 3 and Figure 3.

\section{Discussion}

The theory of Miao medicine classifies diseases with acute 

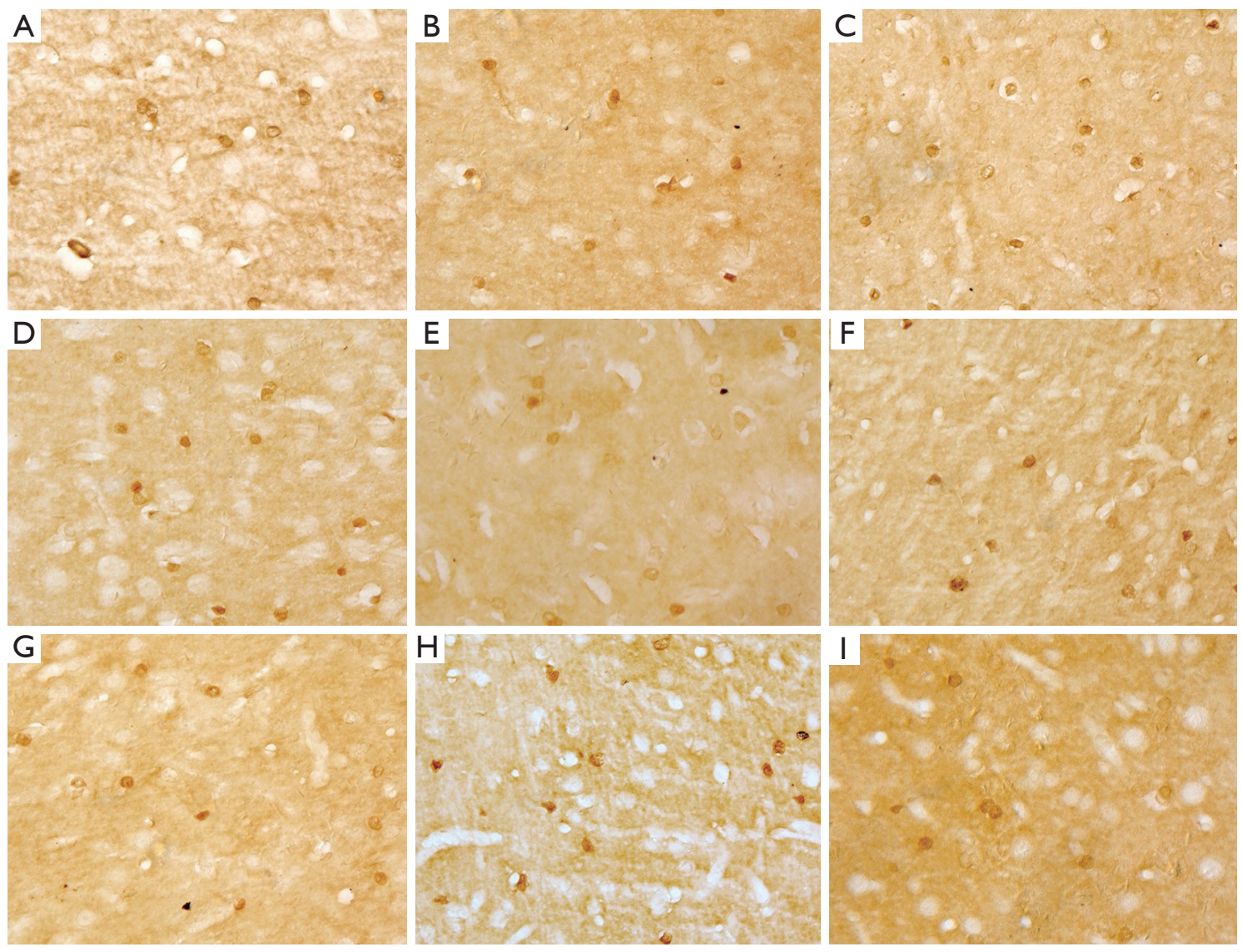

Figure 2 VEGF expression in (A,B,C,D) the frontal lobe and (E,F,G,H,I) the cerebellum of the rats in all groups (immunohistochemistry, ×400). (A,E,I) Sham operation group; (B,F) model group; (C,G) S. miltiorrbiza group; (D,H) Miao medicine group. VEGF, vascular endothelial growth factor; S. miltiorrbiza, salvia miltiorrbiza.

Table 3 EphB2 (number/ $/ \mathrm{mm}^{2}$ ) expression in the frontal lobe and cerebellum of rats in all groups $(\bar{x} \pm \mathrm{s})$

\begin{tabular}{lll}
\hline Groups & Frontal lobe & Cerebellum \\
\hline Sham operation group $(n=8)$ & $72.73 \pm 6.712$ & $37.75 \pm 6.580$ \\
Model group $(n=8)$ & $69.29 \pm 9.163^{\star}$ & $29.88 \pm 7.661^{\star}$ \\
S. miltiorrhiza group $(n=8)$ & $80.48 \pm 7.684^{\text {*\# }}$ & $56.88 \pm 5.566^{\text {*\# }}$ \\
Miao medicine group $(n=8)$ & $102.42 \pm 5.580^{* \# \Delta}$ & $61.00 \pm 9.071^{\text {*\# }}$ \\
\hline
\end{tabular}

Compared with the sham operation group, * $\mathrm{P}<0.05$; compared with the model group, \#, $\mathrm{P}<0.05$; compared with the $S$. miltiorrhiza group, ${ }^{\Delta}, \mathrm{P}<0.05$. EphB2, ephrin-B2; S. miltiorrhiza, salvia miltiorrhiza.

onset, dangerous condition, convulsion, coma, and other symptoms similar to stroke as meridian syndrome. Miao doctors ignore that "tendon is airway and pulse is blood path". When tendons are damaged, Qi will be blocked, and the normal blood circulation will be difficult to promote, leading to blood stasis. Therefore, the principal treatment for impotence and weakness, dyskinesia or numbness, and other pathological changes of the limbs in Miao medicine involves promoting blood circulation and removing blood stasis. Tongqiao Huashuan Decoction, which was prepared by Professor Zhu Guangqi on the basis of folk collection and clinical observation in Miao area, is an effective prescription of Miao medicine for treating apoplexy. It consists of sargentgloryvine stem, spinyleaf pricklyash root, birudo, aleppo dock rbizome, rbizoma cibotii, rbizoma calami, gastrodiae rbizoma, and astragalus membranaceus. Sargentgloryvine stem, one of the four blood-circulatingpromotors of Miao medicine, promotes blood circulation, eliminates wind, and removes dampness. It also inhibits platelet aggregation and directly dilates the coronary artery. Its components include lignin, which has strong antioxidant 


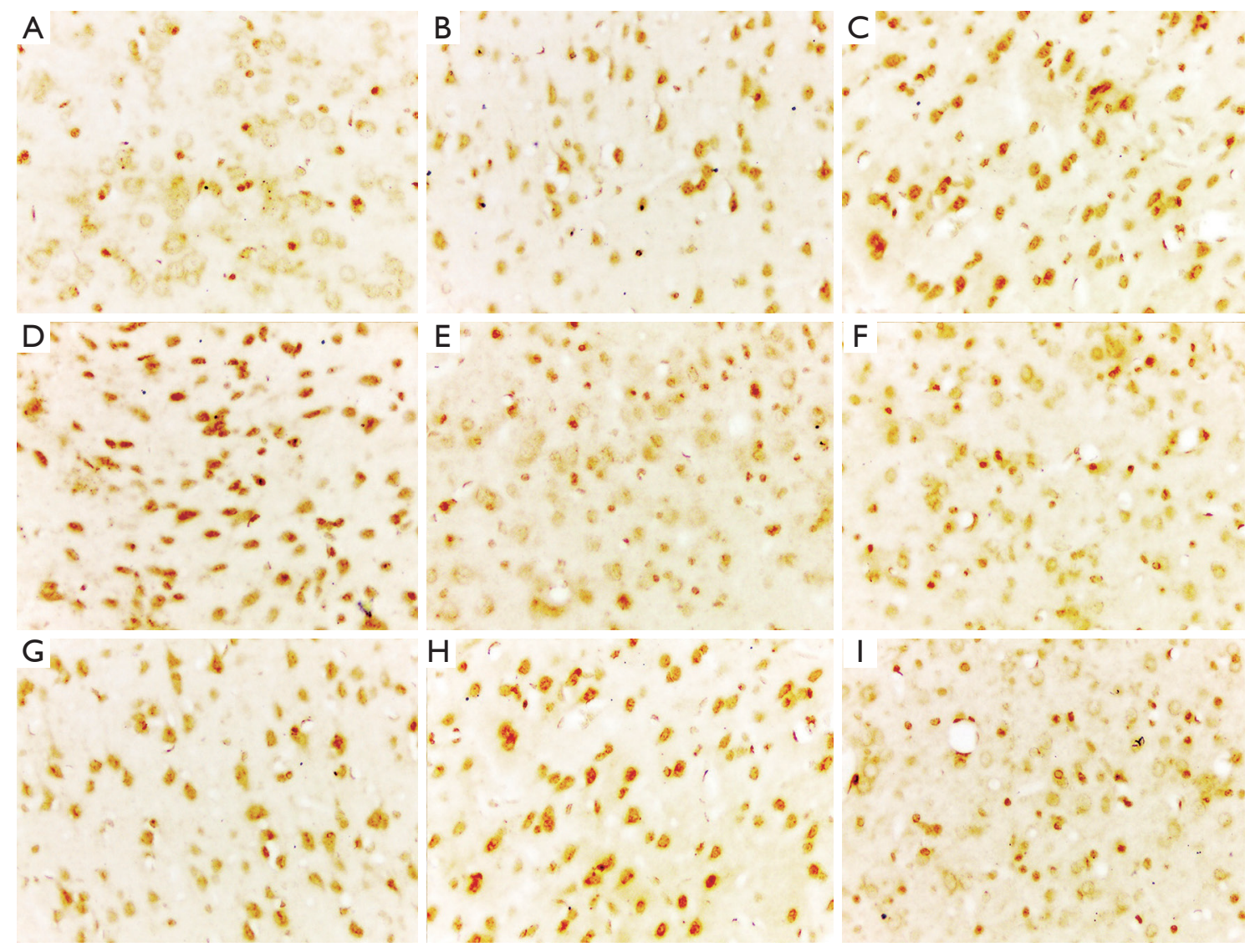

Figure 3 EphB2 expression in (A,B,C,D) the frontal lobe and (E,F,G,H,I) the cerebellum of the rats in all groups (immunohistochemistry, ×400). (A,E,I) Sham operation group; (B,F) model group; (C,G) S. miltiorrbiza group; (D,H) Miao medicine group. EphB2, ephrin-B2; S. miltiorrbiza, salvia miltiorrbiza.

effect, and polysaccharide, which exhibit antioxidant and free radical scavenging effects $(12,13)$. Spinyleaf pricklyash root is also one of the four blood-circulating-promotors of Miao medicine and has the effect of dispelling wind and cold, dispersing blood stasis, dredging collaterals, and relieving pain. Its extract has directly dilates blood vessels, inhibits calcium influx, and serves as calcium antagonist (14). Both sargentgloryvine stem and spinyleaf pricklyash root in the prescription eliminate wind and promote blood circulation. Aleppo dock rbizome, also known as amplexicaule knotweed rhizome, is the whole herb of aleppo dock rbizome, a dicotyledon plant that activates and removes blood stasis. Hirudo can remove blood stasis and activate channels and collaterals. Aleppo dock rbizome and birudo are also minister drugs in the prescription and share the effect of dispersing blood stasis and breaking blood accumulation. Furthermore, they effectively cooperate with monarch drugs. Rbizoma cibotii, rbizoma calami, and gastrodiae rbizoma are all adjuvants. Rhizoma cibotii is good at tonifying the liver and kidney, strengthening muscles and bones, relaxing meridians and collaterals, dispelling wind, and dredging collaterals. Rbizoma calami has a strong and unique bitter taste and mildness in quality, eliminates phlegm, and invigorates the spleen. Gastrodiae rbizoma is a genuine medicine in Guizhou that can calm the liver, quench wind, and increase cerebral blood flow. Astragalus membranaceus is a compound introduced in the prescription. It promotes the Qi and blood circulating activity of all of the drugs in the prescription. All of the drugs are combined to expel wind, dredge collaterals, promote circulation, and remove blood stasis.

After ischemic stroke, ischemic injuries include necrosis of acute infarct central area, the occurrence of ischemia penumbra around the cerebral infarcted area, delayed brain 
injury after infarction, reperfusion injury after infarction, and distant dysfunction. After ischemia, the blood supply of brain tissue is damaged locally, and the structure of its related parts is affected (15). The increase in capillary density, microangiogenesis and collateral circulation in the ischemic area and its surrounding tissues after cerebral infarction are helpful to improve local blood supply and are directly related to the functional outcome and survival time of patients $(1,2)$. The increase in nerve growth inhibitor, decrease in regional cerebral blood flow, stimulation of inflammation, and imbalance of neurotransmitter regulation may be involved in the secondary injury of distant sites after cerebral infarction $(16,17)$. VEGF is a highly specific mitogen of endothelial cells and can act on cortical neurons (18) through various signaling pathways. This study showed that VEGF expression in the right frontal lobe and cerebellum of rats was significantly higher than that in the sham operation group $(\mathrm{P}<0.05)$, suggesting that VEGF expression was increased after cerebral infarction. Microvascularization is the growth of new microvasculature by budding from the original blood vessels, which is the main mechanism of compensation for cerebral ischemia and hypoxia. The increase in local vascular density is consistent with that in VEGF after stroke. However, at this stage, new small vessels are usually composed of immature endothelial cells, and the mature matrix of the cells around the vascular wall is less than that of normal mature vessels. Although immature small vessels can increase the number of blood vessels, the local cerebral blood flow is still reduced. Drugs that can promote angiogenesis can promote not only the formation of small vessels but also the formation of mature blood vessels, thus improving cerebral blood flow and contributing to tissue recovery after cerebral ischemia. The results of our study show that VEGF expression in the frontal lobe and cerebellum of MCAO involved side of the rats is higher in the Miao medicine and S. miltiorrbiza groups than that in the model group and significantly higher in the Miao medicine group than that in the $S$. miltiorrbiza group $(\mathrm{P}<0.05)$. Tongqiao Huashuan Decoction can increase VEGF expression in the frontal lobe and cerebellum of the MCAO-involved side of rats and promote angiogenesis. EphB2 is an important regulator of VEGF signaling pathway. Interaction between endothelial cells and the surrounding mesenchymal cells requires signal transduction between EphB2 and EphB2 receptors. EphB2 is closely related to its ligand and vascular development and is an important regulator of arteriovenous boundary formation, angiogenesis, and germination (3). The growth and infiltration of into the infarct area and its surrounding areas of new blood vessels after cerebral infarction are necessary prerequisites for removing necrotic tissue and tissue repair. EphB2 may also participate in the regulation of blood vessels in distant parts of cerebral infarction. EphB2 signaling pathway shows a bi-directional regulation trend. EphB2 expression shows a downward trend on the $3^{\text {rd }}$ day of cerebral infarction, begins to rise after 2 weeks, generally reaches a peak level on the $21^{\text {st }}$ day, and returns to normal level at approximately 4 weeks. These features may be related to early apoptosis and inhibition of neuronal activity caused by cerebral infarction, and the increase in cerebral cortex neurons, synaptic regeneration, and synaptic formation occurs after 7 days of cerebral infarction. The results of this study indicate a significant difference in EphB2 expression between the frontal lobe in the Miao medicine group and S. miltiorrbiza group $(\mathrm{P}<0.05)$, showing that Miao medicine is superior to $S$. miltiorrbiza. Tongqiao Huashuan Decoction may regulate EphB2 signaling pathway in the frontal lobe and promote its expression in the early stage of cerebral infarction. Given the improvement of neurological function score in rats after treatment, Tongqiao Huashuan Decoction may promote the excitation pathway of cortical neurons' activity and synaptic regeneration, thereby promoting the recovery of nervous function. The results of this study indicate no significant difference in EphB2 expression in the cerebellum between the Miao medicine and S. miltiorrbiza groups $(\mathrm{P}>0.05)$, which may be related to the location of EphB2 expression. EphB2 is generally expressed in the subventricular and cerebral cortex but less in the cerebellum.

In conclusion, Tongqiao Huashuan Decoction can up-regulate EphB2 and VEGF expression in the frontal lobe of the MCAO-involved side of rats and is more effective than S. miltiorrbiza $(\mathrm{P}<0.05)$. Tongqiao Huashuan Decoction may simultaneously regulate EphB2 and VEGF expression in the frontal lobe of MCAO-involved side of rats, promote vascular endothelial cell formation and synaptic regeneration, and thus improve the neurological function of MCAO rats. The mechanism of Tongqiao Huashuan Decoction in improving neurological deficits in MCAO rats may be related to its involvement in regulating VEGF and EphB2 expression at the distal parts of the lesion. 


\section{Acknowledgments}

None.

\section{Footnote}

Conflicts of Interest: The authors have no conflicts of interest to declare.

Ethical Statement: The authors are accountable for all aspects of the work in ensuring that questions related to the accuracy or integrity of any part of the work are appropriately investigated and resolved. The experiment was approved by Animal care welfare committee of Guizhou University of traditional Chinese Medicine (No. 20190001).

\section{References}

1. Schroeter M, Zickler P, Denhardt DT, et al. Increased thalamic neurodegeneration following ischaemic cortical stroke in osteopontin-deficient mice. Brain 2006;129:1426-37.

2. van der Zijden JP, Wu O, van der Toorn A, et al. Changes in neuronal connectivity after stroke in rats as studied by serial manganese-enhanced MRI. Neuroimage 2007;34:1650-7.

3. Grunewald M, Avraham I, Dor Y, et al. VEGF-induced adult neovascularization: recruitment, retention, and role of accessory cells. Cell 2006;124:175-89.

4. Wang J, Wang J, Yu J, et al. Serum VEGF during chemo-radiotherapy and its clinical significance in esophageal squamous cell carcinoma. Transl Cancer Res 2018;7:1199-208.

5. Byrne AM, Bouchier-Hayes DJ, Harmey JH. Angiogenic and cell survival functions of vascular endothelial growth factor (VEGF). J Cell Mol Med 2005;9:777-94.

6. Alexander M, Halmos B. VEGF inhibitors in EGFRmutated lung cancer: a never-ending story? Ann Transl Med 2018;6:446.

7. Foo SS, Turner CJ, Adams S, et al. Ephrin-B2 controls cell motility and adhesion during blood-vessel-wall assembly. Cell 2006;124:161-73.

Cite this article as: Cai J, Wu C, Tang C, Cai J, Cao P, Zhu G. The influence of Tongqiao Huashuan Decoction on the expression of VEGF and EphB2 in MCAO model rats. Ann Palliat Med 2019;8(5):667-675. doi: 10.21037/apm.2019.11.09
8. Tang C, Zhu G, Cai J, et al. The Influence of Tongqiao Huashuan Decoction of Miao medicine on VEGF expression of cerebral infarction area in acute stage. Henan Traditional Chinese Medicine 2016;36:596-9.

9. Cai J, Zhu G, Wang M, et al. The effect of Miao Medicine Tongqiao Huashuan Decoction on ET and NO in acute cerebral infarction patients. Chinese Journal of Integrative Medicine on Cardio-Cerebrovascular Disease 2015;13:2051-4.

10. Zhu G, Wu Y, Hu X, et al. The influence of Miao Medicine Tongqiao Huashuan Decoction on the ultrastructure in cerebral ischemia reperfusion model rats. Chinese Journal of Experimental Traditional Medical Formulae 2013;19:212-5.

11. Longa EZ, Weinstein PR, Carlson S, et al. Reversible middle cerebral artery occlusion without craniectomy in rats. Stroke 1989;20:84-91.

12. Mao S, Gu Q, Cui C, et al. Phenolic compounds from Sargentodoxa cuneate (Oliv.) Rehd et Wils. and their antitumor activities. Chinese Journal of Medicinal Chemistry 2004;14:326-30.

13. Ni S, Fu C, Wu P. Progress in medical studies of Sargentodoxa cuneata (Oliv.) Rehd et Wils. Chinese Wild Plant Resources 2004;23:8-10.

14. Ren X. Dragons blood extract's expansion and the principle of a preliminary study on the blood vessels of the. Journal of Jinan University 1990;11:29-35.

15. Hou Q, Zeng J. Ephrin B2 receptor on the cerebral cortex infarction hematogenously angiogenesis and the impact of neural plasticity. International Journal of Cerebrovascular Disease 2007;15:790-2.

16. Xiong L, Zeng J. Advance in research on secondary damage remote from middle cerebral artery infarction. International Journal of Cerebrovascular Diseases 2005;13:835-8.

17. He Y, Zeng J, Yu J, et al. EphB2-Fc fusion protein promotes the activation of endogenous neural stem cells after experimental cerebral cortical infarction. Chinese Journal of Medicine 2005;85:2395-9.

18. Cébe-Suarez S, Zehnder-Fjällman A, Ballmer-Hofer K. The role of VEGF receptors in angiogenesis; complex partnerships. Cell Mol Life Sci 2006;63:601-15. 